\title{
Jan Grootaers, Actes et acteurs à Vatican II
}

Louvain, Leuven University "Press et Uitgeverij Peeters/Leuven, 1998, 602 p. (préface d'Étienne Fouilloux) (bibliogr., index)

Jean-Louis Schlegel

\section{OpenEdition}

\section{Journals}

Édition électronique

URL : http://journals.openedition.org/assr/470

DOI : $10.4000 /$ assr.470

ISSN : $1777-5825$

Éditeur

Éditions de l'EHESS

\section{Édition imprimée}

Date de publication : 2 octobre 2001

Pagination : $93-156$

ISBN : 2-222-96712-0

ISSN : 0335-5985

Référence électronique

Jean-Louis Schlegel, « Jan Grootaers, Actes et acteurs à Vatican II 》, Archives de sciences sociales des religions [En ligne], 116 | octobre - décembre 2001, document 116.23, mis en ligne le 11 octobre 2005, consulté le 22 septembre 2020. URL : http://journals.openedition.org/assr/470 ; DOI : https://doi.org/ 10.4000/assr.470 
1'action du LGCM a porté principalement sur la revendication de l'accès des homosexuels à l'ordination ou de la non-expulsion des ordres de ceux dont l'homosexualité était rendue publique, et sur la reconnaissance officielle des mariages religieux des couples de même sexe. Sur ce dernier point, si l'on suit l'A., le LGCM aurait, dès la fin des années 1970, inscrit au centre de son action la reconnaissance des unions de personnes de même sexe, notamment en tenant une liste de prêtres et de pasteurs acceptant de réaliser une cérémonie, ou en diffusant des guides de célébration. Les mouvements plus ou moins semblables des États-Unis n'embrassèrent au contraire cette cause qu'à partir du milieu des années quatre-vingt dix.

La troisième partie de l'ouvrage contient trois essais. Celui de S.G. porte sur la théologie gay et lesbienne dans le " contexte postmoderne ». L'essai d'Elizabeth Stuart, professeur de théologie chrétienne, est une critique directe du travail du LGCM, trop blanc, trop masculin, trop peu intéressé à révolutionner le fonctionnement de l'« hétéro-église » (hetero-Church, p. 165), cherchant au contraire une intégration au centre. L'essai de Robert Goss, professeur au département de religion de Webster University, St Louis, aux États-Unis, est assez similaire, bien que centré sur les USA : il recommande de passer d'une théologie cherchant à réconcilier les homosexuels avec les Églises à une théologie «queer » qui vise à transformer les Églises, par exemple en reconnaissant la dimension essentiellement religieuse du " gay sex».

Ces trois auteurs se posent in fine la même question : comment être " inclusif », c'est-àdire mettre au centre de l'action du mouvement social et des Églises, les gays, les lesbiennes, mais aussi les bisexuels, les transgenres (transgender, d'où l'expression «translesbigay movement »), et enfin les hétérosexuels, tout en gardant une unité de revendication.

\section{Baptiste Coulmont.}

\subsection{3}

\section{GROOTAERS (Jan).}

Actes et acteurs à Vatican II, Louvain, Leuven University "Press et Uitgeverij Peeters/Leuven, 1998, 602 p. (préface d'Étienne Fouilloux) (bibliogr., index).

C'est à la fois comme témoin, chroniqueur et historien, pour reprendre les termes du préfacier, que l'auteur s'exprime sur le concile Vatican II et, souvent, sur ses coulisses et ses épisodes obscurs. En tout cas, il l'aborde avec une information de première main, souvent inédite et neuve, en traitant principalement quatre facettes : le rôle des papes et futurs papes, la place de l'opposition, l'importance d'acteurs majeurs, venus de l'Église universelle d'une part, de Belgique et des Pays-Bas d'autre part. Il évoque à la fois des événements et des péripéties, des tendances et des groupes, des figures et des individualités marquantes. La dispersion ou plutôt la richesse et la diversité même des sujets et des hommes dont parle l'A. rendent impossible un compte-rendu quelque peu synthétique. On mettra donc en avant seulement quelques aspects saillants du livre. Pour les papes, on notera en particulier l'influence décisive des interventions de Paul VI dans le vote du texte capital sur la liberté religieuse - malgré le souci de donner quelques gages à la minorité très active et intransigeante opposée à ce texte (on sait le rôle qu'il joua dans l'évolution de Mgr Lefebvre vers l'intégrisme et finalement le schisme). Très intéressante est l'étude précise consacrée aux interventions de Karol Wojtyla, futur Jean-Paul II. L'A. montre bien comment un jeune évêque inconnu s'impose assez rapidement comme une figure essentielle de l'épiscopat polonais (nonobstant, semblet-il, une certaine réserve du cardinal Wyszynski) et comme le symbole de certains changements d'attitude face à l'État communiste. L'insistance dès ce moment-là, chez le jeune Wojtyla, sur la notion de personne comme maillon faible du communisme est remarquable, en même temps que la place centrale constamment accordée au rôle régulateur de l'autorité dans l'Église. Il arrive également au futur pape de conserver un langage théologique particulièrement traditionnel à propos de la nature de l'Église. Enfin, il est à noter qu'il se retrouve parfois dans un front commun des épiscopats de l'Est européen et du tiers-monde face aux évêques «leaders» de l'Europe développée et sécularisée. À travers des invitations à des groupes de travail non officiels, ces derniers tentent néanmoins d'entraîner dans une dynamique commune des évêques de tous les continents. Sur ce point, l'A. donne des renseignements inédits jusqu'à présent à travers une étude de la "Conférence des vingt-deux ».

L'opposition à l'évolution très majoritairement « ouverte» du Concile se traduit en 1963 par une tentative pour en accélérer le processus. Paul VI, qui subit la vive pression des « conservateurs » encore en place à la direction du Concile, utilise habilement un homme de la majorité pour faire passer l'idée d'une accélération nécessaire et pour éviter une quatrième session, en laissant purement et simplement de côté la discussion de divers textes sensibles. La tentative échouera. Mais dans un autre domaine, celui de 
la doctrine du mariage, des amendements en recul sur la dynamique conciliaire seront introduits par Paul VI lui-même... avant de se retrouver cités quasiment tels quels dans l'encyclique Humanae Vitae, en juillet 1968. À cet égard, le livre confirme plutôt l'image d'un Paul VI intellectuellement acquis au renouveau (sauf peut-être à propos du mariage au sens large) mais pratiquement ballotté et souvent incapable de décisions nettes.

À travers les portraits nuancés des figures emblématiques et des principaux acteurs belges et hollandais, on en apprend également beaucoup. Parmi les premières, on appréciera celles de Mgr Colombo, «éminence grise » de Paul VI, de Mgr Felici, « patron » du Concile tout en faisant partie de la minorité qui tente tout au long de contrer sa dynamique ouverte, de Mgr Wyszynski, « cardinal solitaire » soucieux de rappeler au concile la donne des Églises dans les pays communistes.

Enfin, on n'accusera pas l'A. de chauvinisme quand il montre l'importance et l'influence - effectivement grandes - de théologiens et d'évêques belges et néerlandais au concile. Parmi les premiers, outre celui de G. Philips, l'A. a raison de rappeler le rôle central joué par W. Onclin (à propos de la collégialité) et par A. Dondeyne (à propos du «monde», de la culture et de la morale conjugale). Pour les seconds, il est incontestable que le cardinal Alfrink et Mgr Willebrands (sans oublier le cardinal Suenens) furent des acteurs considérables. La description que l'A. donne de la position et des sentiments du protestant Visser't Hooft, secrétaire général du Conseil Ecuménique des Églises, est également de grand intérêt.

Globalement, le livre est passionnant parce que l'A. sait montrer, en véritable historien, la complexité d'un processus où il n'y a pas simplement une majorité et une minorité, où les même acteurs ne sont pas toujours sur la même ligne, où des « reculs » équilibrent des « avancées », où 1'histoire officielle est constamment «doublée » en coulisses par des interventions, des manœuvres, des conflits et des doutes individuels, avec des répercussions en tous sens. Une très importante bibliographie, un index des noms de personnes et des documents conciliaires ainsi qu'un index chronologique complètent l'ensemble.

Jean-Louis Schlegel.
HARRIS (Fredrick C.).

Something Within. Religion in AfricanAmerican Political Activism. New York-Oxford, 1999, 227 p.

La religion des Noirs américains a souvent été perçue comme profondément conservatrice. E. Franklin Frazier (The Negro Church in America, 1963), par exemple, considérait qu'elle était le principal frein aux progrès économiques et éducatifs de la population noire. Le développement d'un corps de croyances fondées sur 1'accès «post-mortem » au royaume de Dieu aurait entravé tout mouvement d'action dans le monde contre la ségrégation et le racisme. La résistance (et parfois l'opposition) du clergé baptiste au mouvement des Civil Rights sembla confirmer ce point de vue. Confronté à l'inertie socio-politique de l'appareil ecclésial, M. Luther King dut par exemple créer une association de pasteurs baptistes indépendante, la Progressive Baptist Convention.

Le paradoxe est que les principaux défenseurs de la cause des Noirs aux États-Unis étaient aussi inspirés par des croyances religieuses. M. Luther King fondait son activisme politique sur un christianisme relu à la lumière de la doctrine de la contestation non violente de Gandhi. L'idéal de fraternité était mobilisé contre la ségrégation dans le Sud, considérée comme une institution «anti-chrétienne ». De façon beaucoup plus radicale, Elijah Muhammad puis Malcom X s'appuyaient sur une conception très hétérodoxe de l'islam pour promouvoir le nationalisme noir américain visant à la séparation entre Blancs et Noirs.

Tout l'intérêt de l'ouvrage de F.C.H. est d'éclaircir ce paradoxe en montrant comment la religion des Noirs américains stimule leur engagement politique depuis les années 1960. Dans le cas des principales Églises baptistes et méthodistes (qui représentent plus de $80 \%$ des fidèles noirs américains), F.C.H. parle d'une « culture civile d'opposition» ( $\mathrm{p}$. 40). Elle a pour caractéristique d'être à la fois attestataire et contestataire. En effet, ces Églises acceptent les « règles du jeu » politique : elles encouragent les fidèles à voter, soutiennent plus ou moins ouvertement certains candidats, forment des cadres susceptibles de s'engager politiquement. Mais, d'un autre côté, les pasteurs noirs se mobilisent en faveur de programmes de réformes sociales devant aider la minorité noire américaine. Tout en acceptant la forme des institutions politiques américaines, les Églises noires baptistes et méthodistes remettent en question la légitimité des inégalités « raciales » aux États-Unis en les dénonçant comme des injustices. 